PROCEEDINGS OF THE

AMERICAN MATHEMATICAL SOCIETY

Volume 135, Number 8, August 2007, Pages 2497-2504

S 0002-9939(07)08776-X

Article electronically published on March 14, 2007

\title{
ON THE REGULARITY OF THE NEUMANN PROBLEM FOR FREE SURFACES WITH SURFACE TENSION
}

\author{
WALTER CRAIG AND ANA-MARIA MATEI
}

(Communicated by Michael I. Weinstein)

\begin{abstract}
In $1952 \mathrm{H}$. Lewy established that a hydrodynamic free surface which is at least $C^{1}$ in a neighborhood of a point $q$ situated on the free surface is automatically $C^{\omega}$, possibly in a smaller neighborhood of $q$. This local result is an example which preceeds the theory developed by D. Kinderlehrer, L. Nirenberg and J. Spruck (1977-79), proving that in many cases free surfaces cannot have an arbitrary regularity; in particular, there exist $k, \mu$ such that if the surface in question is $C^{k, \mu}$, then automatically is $C^{\omega}$. In this paper we extend their methods to Neumann type problems for free surfaces with surface tension.
\end{abstract}

\section{The EQUATIONS OF FREE SURFACES}

The classical equations of motion of an ideal fluid with a free surface in a stationary configuration are set in a domain $\Omega:=\left\{x=\left(x^{\prime}, x_{n}\right) \in \mathbb{R}^{n} \mid x^{\prime} \in \mathbb{R}^{n-1},-h<\right.$ $\left.x_{n}<\eta\left(x^{\prime}\right)\right\}$, where the free surface is $\Sigma:=\left\{x_{n}=\eta\left(x^{\prime}\right)\right\}$. In Euler coordinates, the velocity of the fluid is given by a vector field $\mathbf{u}(x)$ defined on $\Omega$. Since the fluid is incompressible and irrotational,

$$
\nabla \cdot \mathbf{u}=0, \quad \nabla \wedge \mathbf{u}=0 \quad \text { in } \Omega .
$$

Hence $\mathbf{u}(x)=\nabla \Phi(x)$ for a potential function $\Phi$ satisfying

$$
\Delta \Phi=0 \quad \text { in } \Omega \text {. }
$$

The boundary conditions for $\Phi$ are

$$
N \cdot \nabla \Phi=0 \quad \text { on } \partial \Omega
$$

where $N$ is the unit exterior normal to $\partial \Omega$, and additionally on the free surface $\Sigma$

$$
\frac{1}{2}|\nabla \Phi|^{2}+g \eta=\frac{c^{2}}{2},
$$

where the Bernoulli constant $c / 2$ is given in terms of the speed $c$ of the solution.

It is another common problem to include the effects of the surface tension. In this case, the boundary condition (1.3) is replaced by

$$
\frac{1}{2}|\nabla \Phi|^{2}+g \eta+\sigma H(\Sigma)=\frac{c^{2}}{2}, \quad \sigma>0,
$$

Received by the editors October 4, 2005 and, in revised form, March 20, 2006.

2000 Mathematics Subject Classification. Primary 35J65, 76B15.

This research was supported in part by the Canada Research Chairs Program and the NSERC through grant \# 238452-01.

(C)2007 American Mathematical Society Reverts to public domain 28 years from publication 
where $\sigma>0$ is proportional to the Bond number and $H(\Sigma)$ is the mean curvature of the free surface $\Sigma$ :

$$
H(\Sigma)=-\nabla \cdot\left(\frac{\nabla \eta}{\sqrt{1+|\nabla \eta|^{2}}}\right) .
$$

The aim of this paper is to study the regularity of the free surface $\Sigma$. The type of question that we address is illustrated by a classical result of H. Lewy.

Theorem 1.1 (H. Lewy [8). Let $n=2$ and suppose that $\left(\eta\left(x_{1}\right), \Phi\left(x_{1}, x_{2}\right)\right)$ satisfy (1.1), (1.2), (1.3) and that $\eta \in C^{1}, \Phi \in C^{1}(\Omega \cup \Sigma)$, locally in a neighborhood of a point $q \in \Sigma$. Then in fact $(\eta, \Phi) \in C^{\omega}$ in a possibly smaller neighborhood of $q$.

The result of Theorem 1.1 is sharp, in the sense that there exist solutions of (1.1), (1.2), (1.3) which are Lipschitz continuous but not $C^{1}$. These are the Stokes waves of extremal forms [2], which have a crest of open angle $2 \pi / 3$; indeed they are Lipschitz continuous but not $C^{1}$ at the crest.

During the past few years there has been an interest in free surface problems where the effects of the surface tension are not negligible. In this direction, Lewy's theorem has recently been extended to the case which includes the effects of surface tension.

Theorem 1.2 (A.-M. Matei [9]). Let $n=2$ and suppose that $\left(\eta\left(x_{1}\right), \Phi\left(x_{1}, x_{2}\right)\right)$ satisfy (1.1), (1.2), (1.4) and that $\eta \in C^{2, \mu}, \Phi \in C^{2, \mu}(\Omega \cup \Sigma), 0<\mu<1$, locally in a neighborhood of a point $q \in \Sigma$. Then in fact $(\eta, \Phi) \in C^{\omega}$ in a possibly smaller neighborhood of $q$.

The proof of Theorem 1.2 depends upon the existence of a harmonic conjugate function for $\Phi(x)$, which restricts the result to the two-dimensional case. However, the approach is related to the dimension independent techniques developed in [5] by D. Kinderlehrer and L. Nirenberg, and in [6, 7] by D. Kinderlehrer, L. Nirenberg and J. Spruck. The main contribution of the present paper is to extend the result of Theorem 1.2 to three and higher dimensions.

Theorem 1.3. Let $n \geq 3$ and let $\left(\eta\left(x^{\prime}\right), \Phi\left(x^{\prime}, x_{n}\right)\right)$ be a solution of (1.1), (1.2), (1.4). Suppose that $\eta \in C^{2, \mu}, \Phi \in C^{2, \mu}(\Omega \cup \Sigma), 0<\mu<1$, locally in a neighborhood of a point $q \in \Sigma$. Then $(\eta, \Phi) \in C^{\omega}$, in a possibly smaller neighborhood of $q$.

In fact we are able to prove an a priori regularity result for a more general free surface problem with Neumann boundary conditions:

$$
\begin{cases}\Delta \Phi=0 & \text { in } \Omega, \\ N \cdot \nabla \Phi=0 & \text { on } \Sigma, \\ G(x, \nabla \Phi)+H(\Sigma)=0 & \text { on } \Sigma,\end{cases}
$$

where the function $G(x, p)$ is $C^{k, \mu}, k \geq 2$ (respectively $C^{\omega}$ ) in the variables $(x, p) \in$ $\mathbb{R}^{2 n}$.

Theorem 1.4. Let $n \geq 3$ and suppose that $\left(\eta\left(x^{\prime}\right), \Phi\left(x^{\prime}, x_{n}\right)\right)$ is a solution of (1.5) and that $\eta \in C^{2, \mu}, \Phi \in C^{2, \mu}(\Omega \cup \Sigma), 0<\mu<1$, locally in a neighborhood of a point $q \in \Sigma$. Then $\eta, \Phi \in C^{k+2, \mu}$ (respectively, $\eta, \Phi \in C^{\omega}$ ) in a possibly smaller neighborhood of $q$. 
For the hydrodynamic problem of free surface water waves, the function $G$ is given by

$$
G(x, p)=\frac{1}{\sigma}\left(\frac{1}{2}|p|^{2}+g x_{n}-\frac{c^{2}}{2}\right) .
$$

A preliminary version of our results in Theorems 1.3 and 1.4 has appeared in 3 . There is an interesting difference between the hypotheses for the a priori regularity results in the theory developed in [5, 6, 7] and the ones in Theorems 1.3 and 1.4 . In particular the former references work with a nondegeneracy condition on the solution $\Phi$ at the point in question $q$; this has the form of either $(N \cdot \nabla) \Phi(q) \neq$ 0 (in the case of 'type $p=0$ ') or else $(N \cdot \nabla)^{2} \Phi(q) \neq 0$ (in the case of 'type $\left.p=1^{\prime}\right)$. In contrast, for the above results we do not require a nondegeneracy condition to hold on the solution $\Phi$ at the point $q$. The main idea of our proof is to introduce an auxiliary function which will play the role of the harmonic conjugate in two dimensions, even if in higher dimensions this function does not have all the properties of a real conjugate.

\section{Proof of Theorem 1.4}

Consider the problem (1.5) and suppose that $q \in \Sigma$ such that locally near $q$, the potential $\Phi$ and the free surface $\Sigma$ are of class $C^{2, \mu}$. We construct an auxiliary function $\rho$ which will replace the harmonic conjugate function in the result $[9$ in two dimensions. Let $B_{r}(q)$ be a ball centered at $q$ and radius $r$ and let $\rho(x)$ be a function satisfying

$$
\Delta \rho=0 \quad \text { in } \Omega \cap B_{r}(q)
$$

with boundary conditions

$$
\begin{array}{ll}
\rho=0 & \text { on } \quad \Sigma, \\
\rho=g \leq 0 & \text { on } \quad \partial\left(\Omega \cap B_{r}(q)\right) \backslash \Sigma .
\end{array}
$$

We suppose simply that $g \in C^{\infty}$ and that $g$ is not identically zero on $\partial\left(\Omega \cap B_{r}(q)\right) \backslash$ $\Sigma$. The maximum principle implies that $\rho(x)<0$ in $\Omega \cap B_{r}(q)$, and the Hopf lemma implies that $N \cdot \nabla \rho(x)>0$ on $\Sigma$. Furthermore, $\rho$ is smooth in any smaller neighborhood of $q$ in $\Omega$, and indeed $\rho \in C^{2, \mu}\left(\bar{\Omega} \cap B_{\frac{r}{2}}(q)\right)$.

We perform a change of variables in (1.5) and (2.1), using the function $\rho$ to transform $\Omega$ locally to a subset of a half-space, and the free surface $\Sigma$ into a subset of a hyperplane. More precisely, suppose that $q=0$ and that the tangent space of $\Sigma$ at the point $q$ is $T_{q}(\Sigma)=\left\{\left(x^{\prime}, 0\right) \mid x^{\prime} \in \mathbb{R}^{n-1}\right\}$. In a neighborhood of $q=0$ we consider the partial hodograph transformation with respect to $\rho$ :

$$
x=\left(x_{1}, \ldots, x_{n}\right)=\left(x^{\prime}, x_{n}\right) \mapsto y=\left(x^{\prime}, \rho\right)=\left(y^{\prime}, y_{n}\right) .
$$

This transformation is well defined locally, since the Jacobian of the mapping $x \mapsto$ $\left(x^{\prime}, \rho\right)$ at the point $q$ is

$$
\rho_{x_{n}}(0)=N \cdot \nabla \rho(0)>0 .
$$

Moreover, the transformation locally maps $\Omega \rightarrow U \subseteq\left\{y_{n}<0\right\}$ and $\Sigma \rightarrow S \subseteq\left\{y_{n}=\right.$ $0\}$. Define the inverse transformation $x_{n}=\tau(y)$, which is of class $C^{2, \mu}$ locally in $U \cup S$. This map is the analog of the 'type $\mathrm{p}=0$ ' transformation in the theory of D. Kinderlehrer, L. Nirenberg and J. Spruck [5, 6, 7].

The original problem (1.5) together with (2.1) can be thus transformed into a problem in new dependent and independent variables, posed on a domain such that 
the image of the free boundary $\Sigma$ is a subset of the fixed boundary $\left\{y_{n}=0\right\}$. To see this we first express the derivatives of $\rho(x)$ in terms of the derivatives of $\tau(y)$. We have

$$
\tau\left(x^{\prime}, \rho(x)\right)=x_{n}
$$

hence

$$
\tau_{y_{\alpha}}+\rho_{x_{\alpha}} \tau_{y_{n}}=0, \quad \rho_{x_{n}} \tau_{y_{n}}=1
$$

where the index $\alpha$ runs from 1 to $n-1$. It follows that

$$
\rho_{x_{\alpha}}=-\frac{\tau_{y_{\alpha}}}{\tau_{y_{n}}}, \quad \rho_{x_{n}}=\frac{1}{\tau_{y_{n}}} .
$$

Subsequently define $\Psi(y):=\Phi(x)$. We can express the first order derivatives of $\Phi(x)$ in a similar way,

$$
\Phi_{x_{\alpha}}=\Psi_{y_{\alpha}}+\rho_{x_{\alpha}} \Psi_{y_{n}}, \quad \Phi_{x_{n}}=\rho_{x_{n}} \Psi_{y_{n}},
$$

which immediately yields an expression in the variable $y$, given equation (2.2) for the derivatives of $\rho$. The formulae for the second order derivatives of $\Phi$ follow the same way:

$$
\begin{aligned}
\Phi_{x_{\alpha} x_{\alpha}} & =\Psi_{y_{\alpha} y_{\alpha}}+\rho_{x_{\alpha}}^{2} \Psi_{y_{n} y_{n}}+2 \rho_{x_{\alpha}} \Psi_{y_{\alpha} y_{n}}+\rho_{x_{\alpha} x_{\alpha}} \Psi_{y_{n}}, \\
\Phi_{x_{n} x_{n}} & =\rho_{x_{n}}^{2} \Psi_{y_{n} y_{n}}+\rho_{x_{n} x_{n}} \Psi_{y_{n}} .
\end{aligned}
$$

Laplace's equation for the function $\Phi(x)$ is thus transformed into an elliptic equation for $\Psi(y)$ with coefficients given in terms of $\tau(y)$ :

$$
\begin{aligned}
0=\Delta_{x} \Phi & =\Delta_{y^{\prime}} \Psi+\left|\nabla_{x} \rho\right|^{2} \Psi_{y_{n} y_{n}}+2 \sum_{\alpha<n} \rho_{x_{\alpha}} \Psi_{y_{\alpha} y_{n}} \\
& =\Delta_{y^{\prime}} \Psi+\frac{1+\left|\nabla_{y^{\prime}} \tau\right|^{2}}{\tau_{y_{n}}^{2}} \Psi_{y_{n} y_{n}}-2 \sum_{\alpha<n} \frac{\tau_{y_{\alpha}}}{\tau_{y_{n}}} \Psi_{y_{\alpha} y_{n}} .
\end{aligned}
$$

We are using the notation that $\Delta_{y^{\prime}} \Psi=\sum_{\alpha<n} \Psi_{y_{\alpha} y_{\alpha}}$ and $\left|\nabla_{y^{\prime}} \tau\right|^{2}=\sum_{\alpha<n} \tau_{y_{\alpha}}^{2}$. One computes the effect of the change of variables on Laplace's equation for $\rho$ itself (see [5]):

$$
0=\Delta_{x} \rho=\sum_{\alpha<n}\left(-\left(\frac{\tau_{y_{\alpha}}}{\tau_{y_{n}}}\right)_{y_{\alpha}}+\frac{\tau_{y_{\alpha}}}{\tau_{y_{n}}}\left(\frac{\tau_{y_{\alpha}}}{\tau_{y_{n}}}\right)_{y_{n}}\right)+\frac{1}{\tau_{y_{n}}}\left(\frac{1}{\tau_{y_{n}}}\right)_{y_{n}} .
$$

Proposition 2.1 (D. Kinderlehrer and L. Nirenberg [5]). Given $\tau(y) \in C^{2, \mu}$, the equation (2.4) is elliptic in any region in which $\tau_{y_{n}}$ is bounded away from zero.

The boundary conditions in problem (1.5) must also be transformed into the new coordinates. First, since $\Sigma=\left\{x_{n}=\eta\left(y^{\prime}, 0\right)\right\}$ we have

$$
\begin{aligned}
& N_{y}=\frac{1}{\sqrt{1+\left|\nabla_{y^{\prime}} \tau\right|^{2}}}\left(-\nabla_{y^{\prime}} \tau, 1\right), \\
& H(\Sigma)=\frac{1}{n-1} \sum_{\alpha, \beta<n} \frac{\left(\left(1+\left|\nabla_{y^{\prime}} \tau\right|^{2}\right) \delta_{\alpha \beta}-\tau_{y_{\alpha}} \tau_{y_{\beta}}\right) \tau_{y_{\alpha} y_{\beta}}}{\left(1+\left|\nabla_{y^{\prime}} \tau\right|^{2}\right)^{3 / 2}} .
\end{aligned}
$$

The boundary conditions for $\Phi$ on $\Sigma$,

$$
N \cdot \nabla \Phi=0, \quad G(x, \nabla \Phi)+H(\Sigma)=0,
$$

are transformed into

$$
0=N \cdot \nabla \Phi=\frac{1}{\sqrt{1+\left|\nabla_{y^{\prime}} \tau\right|^{2}}}\left(-\sum_{\alpha<n}\left(\Psi_{\alpha}-\frac{\tau_{y_{\alpha}}}{\tau_{y_{n}}} \Psi_{y_{n}}\right) \tau_{y_{\alpha}}+\frac{1}{\tau_{y_{n}}} \Psi_{y_{n}}\right)
$$


respectively

$$
\begin{aligned}
0 & =G(x, \nabla \Phi)+H(\Sigma) \\
& =G\left(\left(y^{\prime}, \tau\right),\left(\nabla_{y^{\prime}} \Psi-\left(\Psi_{y_{n}} / \tau_{y_{n}}\right) \nabla_{y^{\prime}} \tau,\left(1 / \tau_{y_{n}}\right) \Psi_{y_{n}}\right)\right)+H(\Sigma),
\end{aligned}
$$

which are posed on the open subset $S \subset\left\{\left(y^{\prime}, 0\right) \in \mathbb{R}^{n}\right\}$. Define

$$
K\left(y^{\prime}, \tau, \nabla_{y} \tau, \nabla_{y} \Psi\right):=G\left(\left(y^{\prime}, \tau\right),\left(\nabla_{y^{\prime}} \Psi-\frac{\Psi_{y_{n}}}{\tau_{y_{n}}} \nabla_{y^{\prime}} \tau, \frac{1}{\tau_{y_{n}}} \Psi_{y_{n}}\right)\right) .
$$

Combining (2.3), (2.4), (2.5) and (2.6) we obtain

$$
\begin{cases}\Delta_{y^{\prime}} \Psi+\frac{1+\left|\nabla_{y^{\prime}} \tau\right|^{2}}{\tau_{y_{n}}^{2}} \Psi_{y_{n} y_{n}}-2 \sum_{\alpha<n} \frac{\tau_{y_{\alpha}}}{\tau_{y_{n}}} \Psi_{y_{\alpha} y_{n}}=0 & \text { in } U, \\ \sum_{\alpha<n}\left(-\left(\frac{\tau_{y_{\alpha}}}{\tau_{y_{n}}}\right)_{y_{\alpha}}+\frac{\tau_{y_{\alpha}}}{\tau_{y_{n}}}\left(\frac{\tau_{y_{\alpha}}}{\tau_{y_{n}}}\right)_{y_{n}}\right)+\frac{1}{\tau_{y_{n}}}\left(\frac{1}{\tau_{y_{n}}}\right)_{y_{n}}=0 & \text { in } U, \\ -\sum_{\alpha<n}\left(\Psi_{\alpha}-\frac{\tau_{y_{\alpha}}}{\tau_{y_{n}}} \Psi_{y_{n}}\right) \tau_{y_{\alpha}}+\frac{1}{\tau_{y_{n}}} \Psi_{y_{n}}=0 & \text { on } S, \\ K\left(y^{\prime}, \tau, \nabla_{y} \tau, \nabla_{y} \Psi\right)+\frac{1}{n-1} \sum_{\alpha, \beta<n} \frac{\left(\left(1+\left|\nabla_{y^{\prime}} \tau\right|^{2}\right) \delta_{\alpha \beta}-\tau_{y_{\alpha}} \tau_{y_{\beta}}\right) \tau_{y_{\alpha} y_{\beta}}}{\left(1+\left|\nabla_{y^{\prime}} \tau\right|^{2}\right)^{\frac{3}{2}}}=0 & \text { on } S .\end{cases}
$$

Theorem 2.2. Problem (2.7) is elliptic and coercive.

Proof of Theorem 2.2. We have performed a change of coordinates such that $q \rightarrow 0$ and $\Sigma \rightarrow S \subset\left\{\left(y^{\prime}, 0\right), y^{\prime} \in \mathbb{R}^{n-1}\right\}$. We may also assume that the tangent space $\left.T_{q}(\Sigma)=\left\{\left(x^{\prime}, 0\right) \mid x^{\prime} \in \mathbb{R}^{n-1}\right)\right\}$ and that $\rho_{x_{n}}(0)=N \cdot \nabla \rho(0)=1$. Then $\tau_{y_{n}}(0)=1$ and $\nabla_{y^{\prime}} \tau=\mathbf{0}, \Psi_{y_{n}}(0)=\Phi_{x_{n}}(0)=0$. For a comprehensive and concise presentation of ellipticity and coercivity for nonlinear systems we refer the reader to [6]. Since we work locally, and the properties of ellipticity and coercivity are open conditions, it suffices to verify them only at the origin. We will use the notation and the system of weights introduced in [1] and used throughout [5], 6], 7]. Let $s_{1}, s_{2}$ denote the weights of the two equations, $t_{1}, t_{2}$ the weights of $\Psi$, respectively $\tau$, and $r_{1}, r_{2}$ the weights of the two boundary conditions. We choose these weights as follows: $s_{1}=s_{2}=0, t_{1}=t_{2}=2, r_{1}=-1, r_{2}=0$.

The principal part of the system, obtained by linearizing (2.7) at the origin for the variations $\widetilde{\Psi}=\delta \Psi$ and $\widetilde{\tau}=\delta \tau$, takes the form

$$
\begin{cases}\Delta \widetilde{\Psi}=0 & \text { in } U, \\ \Delta \widetilde{\tau}=0 & \text { in } U, \\ -\nabla_{y^{\prime}} \Psi(0) \cdot \nabla_{y^{\prime}} \widetilde{\tau}+\widetilde{\Psi}_{y_{n}}=0 & \text { on } S, \\ \Delta_{y^{\prime}} \widetilde{\tau}=0 & \text { on } S .\end{cases}
$$

The nonlinear problem (2.7) is elliptic and coercive if the linear homogenous problem (2.8) is elliptic and has no nontrivial exponential solution of the form $\widetilde{\tau}=$ $e^{i \xi^{\prime} \cdot y^{\prime}} \bar{\tau}\left(y_{n}\right), \widetilde{\Psi}=e^{i \xi^{\prime} \cdot y^{\prime}} \bar{\Psi}\left(y_{n}\right)$ (with $\xi^{\prime} \in \mathbb{R}^{n-1} \backslash\{0\}$ ) which decay as $y_{n} \rightarrow-\infty$. The linear system (2.8) is clearly elliptic in the domain $U$. To check coercivity we substitute the above form of solutions for $\widetilde{\Psi}, \widetilde{\tau}$ into (2.8) and obtain

$$
\begin{cases}-\left|\xi^{\prime}\right|^{2} \bar{\tau}+\bar{\tau}^{\prime \prime}=0 & \text { in } y_{n} \geq 0 \\ -\left|\xi^{\prime}\right|^{2} \bar{\Psi}+\bar{\Psi}^{\prime \prime}=0 & \text { in } y_{n} \geq 0 \\ -\mathrm{i} \bar{\tau}(0)\left(\nabla_{y^{\prime}} \Psi(0) \cdot \xi^{\prime}\right)+\bar{\Psi}^{\prime}(0)=0, & \\ \bar{\tau}(0)=0 & \end{cases}
$$


The general solution for this problem is $\bar{\tau}\left(y_{n}\right)=A e^{-\left|\xi^{\prime}\right| y_{n}}+B e^{\left|\xi^{\prime}\right| y_{n}}, \bar{\Psi}\left(y_{n}\right)=$ $C e^{-\left|\xi^{\prime}\right| y_{n}}+D e^{\left|\xi^{\prime}\right| y_{n}}$. Given that we only consider solutions which decay as $y_{n} \rightarrow$ $-\infty$, we obtain $A=0, C=0$. The second boundary condition $\bar{\tau}(0)=0$ implies $B=0$, i.e. $\bar{\tau}=\widetilde{\tau}=0$. Replacing $\bar{\tau}=0$ in the first boundary condition we obtain $\bar{\Psi}^{\prime}(0)=0$ which in turn implies $D=0$. This is to say that $\bar{\Psi}=\widetilde{\Psi}=0$.

To complete the proof of Theorem 1.4 we apply the classical regularity theory for nonlinear elliptic systems [10. To do this we initially need to gain one derivative of regularity for $\tau$ and $\Psi$; this can be done by splitting (2.7) into two problems.

$$
\begin{cases}\sum_{\alpha<n}\left(-\left(\frac{\tau_{y_{\alpha}}}{\tau_{y_{n}}}\right)_{y_{\alpha}}+\frac{\tau_{y_{\alpha}}}{\tau_{y_{n}}}\left(\frac{\tau_{y_{\alpha}}}{\tau_{y_{n}}}\right)_{y_{n}}\right)+\frac{1}{\tau_{y_{n}}}\left(\frac{1}{\tau_{y_{n}}}\right)_{y_{n}}=0 & \text { in } U, \\ K\left(y^{\prime}, \tau, \nabla \tau, \nabla \Psi\right)+\frac{1}{n-1} \sum_{\alpha, \beta<n} \frac{\left(\left(1+\left|\nabla_{y^{\prime}} \tau\right|^{2}\right) \delta_{\alpha \beta}-\tau_{y_{\alpha}} \tau_{y_{\beta}}\right) \tau_{y_{\alpha} y_{\beta}}}{\left(1+\left|\nabla_{y^{\prime}} \tau\right|^{2}\right)^{\frac{3}{2}}}=0 & \text { on } S .\end{cases}
$$

Problem (2.9) is elliptic and coercive as discussed above. Recall that $\tau$ is of class $C^{2, \mu}$ locally in $U \cup S$ and since $\Phi$ is of class $C^{2, \mu}$, we have that $\Psi$ is also of class $C^{2, \mu}$ locally in $U \cup S$. In particular, the coefficients of the boundary condition in problem (2.9) are $C^{1, \mu}$. We can now apply Theorem 11.1 in [1]; with the notations in [1] we have $m=1, m_{j}=2$ and $l_{0}=\max \left(m, m_{j}\right)=2$. For $l=3$ the hypotheses of this theorem are satisfied and we obtain that in fact $\tau \in C^{3, \mu}$

Next consider the problem for $\Psi$ with coefficients in terms of $\tau$ :

$$
\begin{cases}\sum_{\alpha<n} \Psi_{y_{\alpha} y_{\alpha}}+\frac{1+\left|\nabla_{y^{\prime}} \tau\right|^{2}}{\tau_{y_{n}}^{2}} \Psi_{y_{n} y_{n}}-2 \sum_{\alpha<n} \frac{\tau_{y_{\alpha}}}{\tau_{y_{n}}} \Psi_{y_{n} y_{\alpha}}=0 & \text { in } U, \\ -\sum_{\alpha<n}\left(\Psi_{y_{\alpha}}-\frac{\tau_{y_{\alpha}}}{\tau_{y_{n}}} \Psi_{y_{n}}\right) \tau_{y_{\alpha}}+\frac{1}{\tau_{y_{n}}} \Psi_{y_{n}}=0 & \text { on } S .\end{cases}
$$

Problem (2.10) is also elliptic and coercive. Since $\tau$ is $C^{3, \mu}$, the coefficients are $C^{2, \mu}$. This time we have $m=1, m_{j}=1$ and $l_{0}=1$. For $l=3$ the hypotheses of Theorem 11.1 in [1] are verified, and we obtain that $\Psi \in C^{3, \mu}$.

Now return to problem (2.7). Over a domain in which $\tau_{y_{n}}$ is bounded away from zero, the function $K$ has the same regularity as $G$ as a function of its arguments, and Theorem 6.8.2 in 10 implies that $\Psi, \tau$ are in fact $C^{k+2, \mu}$, respectively $C^{\omega}$, in $y_{n} \leq 0$ near the origin. More precisely (using the notation of Theorem 6.8.2 of 10]) we have $s_{1}=s_{2}=0, t_{1}=t_{2}=2, h_{1}=r_{1}=-1, h_{2}=r_{2}=0$, and therefore $h_{0}=2$. Since the coefficients of the equations and of the first boundary condition in (2.7) are constant and the domain $U$ is locally a half-plane, the hypotheses on their regularity are automatically verified.

On the other hand since $\tau \in C^{t_{1}+h_{0}-1, \mu}=C^{3, \mu}, \Psi \in C^{t_{2}+h_{0}-1, \mu}=C^{3, \mu}$ and the second boundary condition in (2.7) is $C^{h_{0}+h_{2}, \mu}=C^{2, \mu}$ (recall that $k \geq 2$ ), the last statement in Theorem 6.8.2 implies that $\tau \in C^{t_{1}+h_{0}, \mu}=C^{4, \mu}$ and $\Psi \in C^{t_{2}+h_{0}, \mu}=$ $C^{4, \mu}$. We can now apply the first part of Theorem 6.8.2. Assume $G$ is $C^{k, \mu}$; if we take $h=k$, the second boundary condition is $C^{h+h_{2}, \mu}=C^{k, \mu}$, and we obtain that in fact $\tau \in C^{t_{1}+h, \mu}=C^{k+2, \mu}$ and $\Psi \in C^{t_{2}+h, \mu}=C^{k+2, \mu}$. This is the conclusion we seek. The analytic case follows in the same manner. Finally, since $\eta\left(x^{\prime}\right)=\tau\left(y^{\prime}, 0\right)$, it follows that $\eta$ and therefore $\Phi, \rho$ are $C^{k+2, \mu}$, respectively $C^{\omega}$, locally near $q$. This ends the proof of the theorem. 


\section{A REMARK ON THE CASE WITHOUT SURFACE TENSION}

Consider the Neumann type free surface problem without surface tension:

$$
\begin{cases}\Delta \Phi=0 & \text { in } \Omega \\ N \cdot \nabla \Phi=0 & \text { on } \Sigma \\ G(x, \nabla \Phi)=0 & \text { on } \Sigma\end{cases}
$$

In dimension $n \geq 3$ there exists a counterexample to a regularity result for (3.1) similar to that of Theorem 1.4 of [5]. Indeed, let

$$
G(x, \nabla \Phi)=|\nabla \Phi|^{2}-1,
$$

and consider the harmonic function $\Phi(x)=x_{1}$. The free boundary will be $\Sigma=$ $\left\{x_{n}=\eta\left(x_{2}, \ldots, x_{n-1}\right)\right\}$. It is immediate that $|\nabla \Phi|=1$ and that since the normal $N$ to the boundary is parallel to $\left(0,-\eta_{x_{2}}, \ldots,-\eta_{x_{n-1}}, 1\right)$ we have $\nabla \Phi=(1,0, \ldots, 0) \perp$ $N$. The function $\Phi$ is therefore a solution of (3.1). However the function $\eta$ may have arbitrary regularity and therefore, a general result as the one in Theorem 1.4 is not possible.

In the case of equation (3.1), if we follow the same reasoning as in the proof of Theorem 1.4 we find that the linearized problem is not coercive. Indeed, the boundary conditions of the linearized problem are

$$
\left(\begin{array}{cc}
\partial_{y_{n}} & -\nabla_{y^{\prime}} \Psi(0) \cdot \nabla_{y^{\prime}} \\
\nabla_{p^{\prime}} G(\zeta) \cdot \nabla_{y^{\prime}}+G_{p_{n}}(\zeta) \partial_{y_{n}} & G_{x_{n}}(\zeta)
\end{array}\right)\left(\begin{array}{c}
\widetilde{\Psi} \\
\widetilde{\tau}
\end{array}\right)=0,
$$

where $\zeta=\left(0, \nabla_{y^{\prime}} \Psi(0), 0\right)$. In particular, for the hydrodynamic problem $G$ is given by

$$
G(x, \nabla \Phi)=\frac{1}{2}|\nabla \Phi|^{2}+\mathrm{g} \cdot x-\frac{c^{2}}{2}
$$

(where the vector $-\mathrm{g}$ indicates the acceleration of gravity), and we have

$\nabla_{p^{\prime}} G\left(0, \nabla_{y^{\prime}} \Psi(0), 0\right)=\nabla_{y^{\prime}} \Psi(0), \quad G_{p_{n}}\left(0, \nabla_{y^{\prime}} \Psi(0), 0\right)=\Psi_{y_{n}}(0)=0, \quad G_{x_{n}}=\mathrm{g}_{x_{n}}$.

It is easy to see that these boundary conditions do not give rise to a coercive problem, as the principal symbol of $(3.2)$ is $\left(\nabla_{y^{\prime}} \Psi(0) \cdot \xi^{\prime}\right)^{2}$, which is degenerate whenever the spatial dimension $n$ is greater than $n=2$. However the total symbol

$$
\mathrm{g}_{x_{n}}\left|\xi^{\prime}\right|-\left(\nabla_{y^{\prime}} \Psi(0) \cdot \xi^{\prime}\right)^{2}
$$

changes sign when $g_{x_{n}}>0$ because of the sign of the subprincipal symbol. This condition on the sign of $g_{x_{n}}$ can be interpreted as the physical condition that at the point $q$, the surface $\Sigma$ is bounding the region of water from above in the considered configuration. This observation indicates that regularity is a global issue, see [6]. This contrasts with the case where the total symbol is positive. We believe that it is very probable that the condition $\operatorname{det}(3.2) \neq 0$ will imply the a priori regularity of the free surface (3.1).

\section{REFERENCES}

[1] S. Agmon, A. Douglis, \& L. Nirenberg. Estimates near the boundary for solutions of elliptic partial differential equations satisfying general boundary conditions: I. Comm. Pure Appl. Math. 12 (1959), 623-727. MR0125307 (23:A2610)

[2] C. J. Amick, L. E. Fraenkel, \& J. F. Toland. On the Stokes conjecture for the wave of extreme form. Acta Matematica 148 (1982), 193 - 214. MR0666110(83m:35147) 
[3] W. Craig \& A.-M. Matei. Sur la régularité des ondes progressives à la surface de l'eau. Journées Equations aux Dérivées Partielles, Exp. No. IV, 9 pp., Univ. Nantes, Nantes, 2003. MR2050590 (2004m:35209)

[4] D. Gilbarg \& N. S. Trudinger. Elliptic Partial Differential Equations of Second Order, Springer Verlag, New York - Berlin, (1977). MR0473443 (57:13109)

[5] D. Kinderlehrer, L. Nirenberg. Regularity in free boundary value problems. Ann. Scuola. Norm. Sup. Pisa, Ser IV, IV (1977), 373-391. MR0440187(55:13066)

[6] D. Kinderlehrer, D. L. Nirenberg \& J. Spruck. Regularity in elliptic free boundary problems I. Journal d'Analyse Math. 34 (1978), 86-119. MR0531272 (83d:35060)

[7] D. Kinderlehrer, L. Nirenberg \& J. Spruck. Regularity in elliptic free boundary problems II: Equations of higher order. Ann. Scuola. Norm. Sup. Pisa, Ser IV VI (1979), 637-683. MR0563338 (83d:35061)

[8] H. Lewy. A note on harmonic functions and a hydrodynamical application. Proc. AMS 3 (1952), 111-113. MR0049399 (14:168c)

[9] A.-M. Matei. The Neumann problem for free boundaries in two dimensions. C.R. Acad. Sci. Paris, Ser. I 335 (7), (2002), 1-6. MR.1941301 (2003i:35286)

[10] C. B. Morrey. Multiple integrals in the calculus of variations. Springer Verlag, New York Berlin, (1966). MR0202511 (34:2380)

Department of Mathematics and Statistics, McMaster University, Hamilton, OnTARIO, CANAda L8S 4K1

E-mail address: craig@math.mcmaster.ca

Department of Mathematics and Computer Science, Loyola University New Orleans, 6363 St. Charles Avenue, New Orleans, Louisiana 70118

E-mail address: amatei@loyno.edu 